\title{
Nonstandard Dirichlet problems with competing $(p, q)$-Laplacian, convection, and convolution
}

\author{
Dumitru Motreanu and Viorica Venera Motreanu
}

Dedicated to Professor Gheorghe Moroşanu on the occasion of his 70th anniversary.

\begin{abstract}
The paper focuses on a nonstandard Dirichlet problem driven by the operator $-\Delta_{p}+\mu \Delta_{q}$, which is a competing $(p, q)$-Laplacian with lack of ellipticity if $\mu>0$, and exhibiting a reaction term in the form of a convection (i.e., it depends on the solution and its gradient) composed with the convolution of the solution with an integrable function. We prove the existence of a generalized solution through a combination of fixed-point approach and approximation. In the case $\mu \leq 0$, we obtain the existence of a weak solution to the respective elliptic problem.
\end{abstract}

Mathematics Subject Classification (2010): 35J92, 47H30.

Keywords: Competing $(p, q)$-Laplacian, Dirichlet problem, convection, convolution, generalized solution, weak solution.

\section{Introduction}

In this paper we consider the following quasilinear problem with homogeneous Dirichlet boundary condition on a bounded domain $\Omega \subset \mathbb{R}^{N}$ with the boundary $\partial \Omega$,

$$
\begin{cases}-\Delta_{p} u+\mu \Delta_{q} u=f(x, \rho * u, \nabla(\rho * u)) & \text { in } \Omega, \\ u=0 & \text { on } \partial \Omega\end{cases}
$$

for $1<q<p<+\infty, \mu \in \mathbb{R}$, and $\rho \in L^{1}\left(\mathbb{R}^{N}\right)$. To ease the exposition we assume $p<N$ mentioning that the complementary case $p \geq N$ can be handled along the same lines.

In order to simplify the notation, for any real number $r>1$, we set $r^{\prime}=r /(r-1)$ (the Hölder conjugate of $r$ ). In particular, we have $p^{\prime}=p /(p-1)<q^{\prime}=q /(q-1)$. In the left-hand side of equation (1.1) there are the negative $p$-Laplacian

$$
-\Delta_{p}: W_{0}^{1, p}(\Omega) \rightarrow W^{-1, p^{\prime}}(\Omega)
$$


expressed as

$$
\left\langle-\Delta_{p} u, v\right\rangle=\int_{\Omega}|\nabla u(x)|^{p-2} \nabla u(x) \cdot \nabla v(x) d x \text { for all } u, v \in W_{0}^{1, p}(\Omega)
$$

and the negative $q$-Laplacian $-\Delta_{q}: W_{0}^{1, q}(\Omega) \rightarrow W^{-1, q^{\prime}}(\Omega)$ expressed as

$$
\left\langle-\Delta_{q} u, v\right\rangle=\int_{\Omega}|\nabla u(x)|^{q-2} \nabla u(x) \cdot \nabla v(x) d x \text { for all } u, v \in W_{0}^{1, q}(\Omega) .
$$

Hereafter, $|\cdot|$ stands for the Euclidean norm in $\mathbb{R}^{N}$. Since $1<q<p<+\infty$, it holds the continuous embedding $W_{0}^{1, p}(\Omega) \hookrightarrow W_{0}^{1, q}(\Omega)$, so the operator $-\Delta_{p}+\mu \Delta_{q}$ is well defined on $W_{0}^{1, p}(\Omega)$. In the sequel, $p^{*}$ stands for the Sobolev critical exponent $p^{*}=N p /(N-p)($ recall that we assume $p<N)$.

The right-hand side of the equation in (1.1) is described by means of a Carathéodory function $f: \Omega \times \mathbb{R} \times \mathbb{R}^{N} \rightarrow \mathbb{R}$ (meaning that $f(\cdot, s, \xi)$ is measurable on $\Omega$ for all $(s, \xi) \in \mathbb{R} \times \mathbb{R}^{N}$ and $f(x, \cdot, \cdot)$ is continuous for a.e. $x \in \Omega$ ) which is composed with the convolution

$$
\rho * u(x)=\int_{\mathbb{R}^{\mathbb{N}}} \rho(x-y) u(y) d y \text { for a.e. } x \in \mathbb{R}^{N}
$$

of some $\rho \in L^{1}\left(\mathbb{R}^{N}\right)$ and $u \in W_{0}^{1, p}(\Omega) \subset W^{1, p}\left(\mathbb{R}^{N}\right)$. Notice that the convolution $\rho * u$ is well defined.

There are two noticeable aspects related to the right-hand side of the equation in (1.1). The first one is the fact that it exhibits dependence not only with respect to the solution $u$ but also with respect to its gradient $\nabla u$. Such a term is usually called convection and its presence prevents us to make use of variational methods. A systematic study of problems with convection can be found in [4]. A second significant feature related to the right-hand side of the equation in (1.1) is the fact that the convection is composed with a convolution which is nonlocal operator. The study of the problems involving the composition of convection and convolution has been started in [6], specifically for problem (1.1) with $\mu \leq 0$. This study incorporates the case where the operator is the $p$-Laplacian $-\Delta_{p}$ (for $\left.\mu=0\right)$ and the ordinary $(p, q)$ Laplacian $-\Delta_{p}-\Delta_{q}$ (for $\mu=-1$ ). The investigation of a (nonsmooth) version of problem (1.1) for an arbitrary $\mu \in \mathbb{R}$, but without convection and convolution, was initiated in [3]. Problem (1.1) with the "competing" $(p, q)$-Laplacian $-\Delta_{p}+\Delta_{q}$ (i.e., in the case where $\mu=1$ ) and convection but without convolution was addressed in $[5]$.

Let $\lambda_{1, p}>0$ denote the first eigenvalue of the negative $p$-Laplacian on $W_{0}^{1, p}(\Omega)$, which is given by the following variational characterization (see, e.g., $[7, \S 9.2]$ ),

$$
\lambda_{1, p}=\min \left\{\frac{\|\nabla u\|_{L^{p}\left(\Omega, \mathbb{R}^{N}\right)}^{p}}{\|u\|_{L^{p}(\Omega)}^{p}}: u \in W_{0}^{1, p}(\Omega) \backslash\{0\}\right\} .
$$

We assume that the following growth condition for $f(x, s, \xi)$ is satisfied. 
Assumption 1.1. There holds

$$
|f(x, s, \xi)| \leq \sigma(x)+a_{1}|s|^{p-1}+a_{2}|\xi|^{p-1}
$$

for a.e. $x \in \Omega$, all $s \in \mathbb{R}$, and $\xi \in \mathbb{R}^{N}$, with a function $\sigma \in L^{r^{\prime}}(\Omega)$ where $r \in\left[1, p^{*}\right)$ and constants $a_{1}, a_{2} \geq 0$ satisfying

$$
\|\rho\|_{L^{1}\left(\mathbb{R}^{N}\right)}^{p-1}\left(a_{1} \lambda_{1, p}^{-1}+a_{2} N^{p-1} \lambda_{1, p}^{-\frac{1}{p}}\right)<1 .
$$

Remark 1.2. The condition (1.4) in Assumption 1.1 can be expressed by saying that the parameter $\rho \in L^{1}\left(\mathbb{R}^{N}\right)$ in problem (1.1) is small enough with respect to its $L^{1}$ norm.

Remark 1.3. (a) If the Carathéodory function $f$ satisfies the growth condition

$$
|f(x, s, \xi)| \leq \sigma(x)+a_{1}|s|^{p-1}+a_{2}|\xi|^{\beta}
$$

as in (1.3) except that the exponent of $|\xi|$ is some $\beta \in[0, p-1)$, then Assumption 1.1 is fulfilled provided that

$$
a_{1}\|\rho\|_{L^{1}\left(\mathbb{R}^{N}\right)}^{p-1}<\lambda_{1, p} .
$$

(b) If $f$ satisfies the stronger growth condition

$$
|f(x, s, \xi)| \leq \sigma(x)+a_{1}|s|^{\alpha}+a_{2}|\xi|^{\beta}
$$

with $\alpha, \beta \in[0, p-1)$, then Assumption 1.1 is fulfilled.

By a generalized solution to problem (1.1) we mean any function $u \in W_{0}^{1, p}(\Omega)$ for which there exists a sequence $\left\{u_{n}\right\}_{n \geq 1}$ in $W_{0}^{1, p}(\Omega)$ such that

(a) $u_{n} \rightarrow u$ in $W_{0}^{1, p}(\Omega)$ as $n \rightarrow \infty$;

(b) $-\Delta_{p} u_{n}+\mu \Delta_{q} u_{n}-f\left(\cdot, \rho * u_{n}(\cdot), \nabla\left(\rho * u_{n}\right)(\cdot)\right) \rightarrow 0$ in $W^{-1, p^{\prime}}(\Omega)$ as $n \rightarrow \infty$;

(c) $\lim _{n \rightarrow \infty}\left\langle-\Delta_{p} u_{n}+\mu \Delta_{q} u_{n}, u_{n}-u\right\rangle=0$.

The essential point in our work is that the driving operator $-\Delta_{p}+\mu \Delta_{q}$ in problem (1.1) has a fundamentally different behavior depending on whether $\mu \leq 0$ or $\mu>0$. Indeed, in the latter case, the operator lacks the ellipticity: notice for instance that, for a nonzero $u_{0} \in W_{0}^{1, p}(\Omega)$ and a number $\lambda>0$, the quantity

$$
\left\langle-\Delta_{p}\left(\lambda u_{0}\right)+\mu \Delta_{q}\left(\lambda u_{0}\right), \lambda u_{0}\right\rangle=\lambda^{p}\left\|\nabla u_{0}\right\|_{L^{p}\left(\Omega, \mathbb{R}^{N}\right)}^{p}-\lambda^{q} \mu\left\|\nabla u_{0}\right\|_{L^{q}\left(\Omega, \mathbb{R}^{N}\right)}^{q}
$$

does not keep a constant sign if $\mu>0$. It is positive for $\lambda>0$ sufficiently large and it is negative for $\lambda>0$ sufficiently small. In view of this, in [3], the operator $-\Delta_{p}+\mu \Delta_{q}$ for $\mu>0$ was called a competing $(p, q)$-Laplacian. Due to the lack of ellipticity there is no available method to handle problem (1.1) for arbitrary $\mu$. In order to bypass this drawback, the notion of generalized solution was introduced in [3] for a counterpart of problem (1.1) without convolution. Note that, in the case where $\mu \leq 0$, the notions of generalized solution and weak solution coincide (see Lemma 3.3). In Theorem 3.4, we prove the existence of a generalized solution to problem (1.1) for arbitrary $\mu$. Our approach relies on a fixed-point theorem and approximation process. Our treatment of problem (1.1) is unified in the sense that it does not distinguish according to the sign of $\mu$. 


\section{Preliminaries}

In the sequel, the space $W_{0}^{1, p}(\Omega)$ is considered endowed with the norm $\|\nabla(\cdot)\|_{L^{p}\left(\Omega, \mathbb{R}^{N}\right)} \cdot$

\subsection{Galerkin basis}

Due to the density of $C_{0}^{\infty}(\Omega)$ in $W_{0}^{1, p}(\Omega)$, the Banach space $W_{0}^{1, p}(\Omega)$ with $1<p<+\infty$ is separable. Therefore, there exists a Galerkin basis of $W_{0}^{1, p}(\Omega)$, that is a sequence $\left\{X_{n}\right\}_{n \geq 1}$ of vector subspaces of $W_{0}^{1, p}(\Omega)$ satisfying

(i) $\operatorname{dim} X_{n}<\infty, \quad \forall n \geq 1$;

(ii) $X_{n} \subset X_{n+1}, \quad \forall n \geq 1$;

(iii) $\overline{\bigcup_{n \geq 1} X_{n}}=W_{0}^{1, p}(\Omega)$.

For the rest of the paper we fix a Galerkin basis $\left\{X_{n}\right\}_{n \geq 1}$ of $W_{0}^{1, p}(\Omega)$.

\subsection{Rellich-Kondrachov theorem}

For $1<p<N$, as known from the Rellich-Kondrachov theorem, the Sobolev space $W_{0}^{1, p}(\Omega)$ is compactly embedded into $L^{\theta}(\Omega)$ if $1 \leq \theta<p^{*}\left(=\frac{N p}{N-p}\right)$ and continuously embedded if $\theta=p^{*}$. For every $\theta \in\left[1, p^{*}\right]$ we denote by $S_{\theta}>0$ the best constant for this embedding, hence

$$
\|u\|_{L^{\theta}(\Omega)} \leq S_{\theta}\|\nabla u\|_{L^{p}\left(\Omega, \mathbb{R}^{N}\right)}, \quad \forall u \in W_{0}^{1, p}(\Omega) .
$$

For $\theta=p$, we have that $S_{p}=\lambda_{1, p}^{-\frac{1}{p}}($ see $(1.2))$.

\subsection{Convolution}

For easy reference we list a few useful properties of the convolution $\rho * u$ of $\rho \in L^{1}\left(\mathbb{R}^{N}\right)$ and $u \in W_{0}^{1, p}(\Omega)$; we refer to $[1, \S 4.4, \S 9.1]$ for details. In order to have well defined the convolution $\rho * u$ of $\rho \in L^{1}\left(\mathbb{R}^{N}\right)$ with $u \in W_{0}^{1, p}(\Omega)$, it is convenient to consider the Sobolev space $W_{0}^{1, p}(\Omega)$ embedded in $W^{1, p}\left(\mathbb{R}^{N}\right)$ by identifying every $u \in W_{0}^{1, p}(\Omega)$ with its extension equal to zero outside $\Omega$. The convolution $\rho * u$ is defined by

$$
\rho * u(x)=\int_{\mathbb{R}^{\mathbb{N}}} \rho(x-y) u(y) d y \text { for a.e. } x \in \mathbb{R}^{N} .
$$

The weak partial derivatives of the convolution $\rho * u$ are expressed by

$$
\frac{\partial}{\partial x_{i}}(\rho * u)=\rho * \frac{\partial u}{\partial x_{i}} \in L^{p}\left(\mathbb{R}^{N}\right), \quad \forall i=1, \ldots, N .
$$

There hold the estimates

$$
\|\rho * u\|_{L^{r}\left(\mathbb{R}^{N}\right)} \leq\|\rho\|_{L^{1}\left(\mathbb{R}^{N}\right)}\|u\|_{L^{r}(\Omega)}
$$

whenever $r \in\left[1, p^{*}\right]$ and

$$
\left\|\rho * \frac{\partial u}{\partial x_{i}}\right\|_{L^{p}\left(\mathbb{R}^{N}\right)} \leq\|\rho\|_{L^{1}\left(\mathbb{R}^{N}\right)}\left\|\frac{\partial u}{\partial x_{i}}\right\|_{L^{p}(\Omega)}, \quad \forall i=1, \ldots, N .
$$


Using the convexity of the function $t \mapsto t^{p}$ on $(0,+\infty)$ and $(2.3)$, we derive that

$$
\begin{aligned}
& \|\nabla(\rho * u)\|_{L^{p}\left(\mathbb{R}^{N}, \mathbb{R}^{N}\right)}^{p}=\int_{\mathbb{R}^{N}}|\nabla(\rho * u)|^{p} d x=\int_{\mathbb{R}^{N}}\left(\sum_{i=1}^{N}\left(\rho * \frac{\partial u}{\partial x_{i}}\right)^{2}\right)^{\frac{p}{2}} d x \\
& \left.\leq \int_{\mathbb{R}^{N}}\left(\sum_{i=1}^{N}\left|\rho * \frac{\partial u}{\partial x_{i}}\right|\right)\right)^{p} d x \leq N^{p-1} \sum_{i=1}^{N}\left\|\rho * \frac{\partial u}{\partial x_{i}}\right\|_{L^{p}\left(\mathbb{R}^{N}\right)}^{p} \\
& \leq N^{p-1}\|\rho\|_{L^{1}\left(\mathbb{R}^{N}\right)}^{p} \sum_{i=1}^{N}\left\|\frac{\partial u}{\partial x_{i}}\right\|_{L^{p}(\Omega)}^{p} \leq N^{p}\|\rho\|_{L^{1}\left(\mathbb{R}^{N}\right)}^{p}\|\nabla u\|_{L^{p}\left(\Omega, \mathbb{R}^{N}\right)}^{p}
\end{aligned}
$$

\subsection{Fixed point theorem}

An essential tool in our approach will be the following consequence of Brouwer's fixed point theorem (see [8, page 37]).

Lemma 2.1. Let $X$ be a finite-dimensional space endowed with the norm $\|\cdot\|_{X}$ and let $A: X \rightarrow X^{*}$ be a continuous mapping. Assume that there is a constant $R>0$ such that

$$
\langle A(v), v\rangle \geq 0 \text { for all } v \in X \text { with }\|v\|_{X}=R \text {. }
$$

Then there exists $u \in X$ with $\|u\|_{X} \leq R$ satisfying $A(u)=0$.

\section{Main result}

In this section we provide our main result regarding the existence of solutions to problem (1.1).

\subsection{Nonlinear operator associated to problem (1.1)}

Hereafter we consider the operator $A: W_{0}^{1, p}(\Omega) \rightarrow W^{-1, p^{\prime}}(\Omega)$ given by

$$
\langle A(u), v\rangle=\left\langle-\Delta_{p} u+\mu \Delta_{q} u, v\right\rangle-\int_{\Omega} f(x, \rho * u(x), \nabla(\rho * u)(x)) v(x) d x
$$

which arises from problem (1.1).

Lemma 3.1. Suppose that (1.3) in Assumption 1.1 is fulfilled. Then, the operator $A: W_{0}^{1, p}(\Omega) \rightarrow W^{-1, p^{\prime}}(\Omega)$ defined in (3.1) is continuous.

Proof. Relations (2.2) and (2.4) imply that the operator $T: W_{0}^{1, p}(\Omega) \rightarrow L^{p}(\Omega) \times$ $L^{p}(\Omega)^{N}$ given by $T(u)=\left(\left.\rho * u\right|_{\Omega},\left.\nabla(\rho * u)\right|_{\Omega}\right)$ is linear and continuous. The growth condition in (1.3) allows to apply the Krasnoselskii theorem [2] which implies that the Nemytskii operator

$$
N_{f}: L^{p}(\Omega) \times L^{p}(\Omega)^{N} \rightarrow L^{p^{\prime}}(\Omega),(v, w) \mapsto f(\cdot, v(\cdot), w(\cdot))
$$

is well defined and continuous. We infer that the operator

$$
W_{0}^{1, p}(\Omega) \rightarrow L^{p^{\prime}}(\Omega), u \mapsto f(\cdot, \rho * u(\cdot), \nabla(\rho * u)(\cdot))
$$

is continuous as the composition of continuous operators. Note also that $L^{p^{\prime}}(\Omega)$ is continuously embedded in $W^{-1, p^{\prime}}(\Omega)$. 
The operators $-\Delta_{p}: W_{0}^{1, p}(\Omega) \rightarrow W^{-1, p^{\prime}}(\Omega)$ and $-\Delta_{q}: W_{0}^{1, q}(\Omega) \rightarrow W^{-1, q^{\prime}}(\Omega)$ are continuous. Since $q<p$ and $\Omega$ is bounded, we have that $W_{0}^{1, p}(\Omega)$ is continuously embedded in $W_{0}^{1, q}(\Omega)$ and $W^{-1, q^{\prime}}(\Omega)$ is continuously embedded in $W^{-1, p^{\prime}}(\Omega)$. Therefore, $-\Delta_{p}+\mu \Delta_{q}: W_{0}^{1, p}(\Omega) \rightarrow W^{-1, p^{\prime}}(\Omega)$ is continuous.

Altogether, this shows that the operator $A$ is continuous.

\subsection{Finite-dimensional approximations}

Given a Galerkin basis $\left\{X_{n}\right\}_{n \geq 1}$ of $W_{0}^{1, p}(\Omega)$, we construct a corresponding sequence of approximate solutions related to problem (1.1).

Proposition 3.2. Suppose that Assumption 1.1 is fulfilled. Then, for every $n \geq 1$, there exists $u_{n} \in X_{n}$ such that

$$
\left\langle-\Delta_{p} u_{n}+\mu \Delta_{q} u_{n}, v\right\rangle=\int_{\Omega} f\left(x, \rho * u_{n}(x), \nabla\left(\rho * u_{n}\right)(x)\right) v(x) d x
$$

for all $v \in X_{n}$. Moreover, the sequence $\left\{u_{n}\right\}_{n \geq 1}$ so obtained is bounded in $W_{0}^{1, p}(\Omega)$.

Proof. On each finite-dimensional space $X_{n}$ we consider the mapping $A_{n}: X_{n} \rightarrow X_{n}^{*}$ defined by

$$
\left\langle A_{n}(u), v\right\rangle=\left\langle-\Delta_{p} u+\mu \Delta_{q} u, v\right\rangle-\int_{\Omega} f(x, \rho * u(x), \nabla(\rho * u)(x)) v(x) d x
$$

for all $u, v \in X_{n}$. Note that $A_{n}$ is continuous (see Lemma 3.1). Our goal is to apply Lemma 2.1 to the operator $A_{n}$. To this end, we note from (1.3) in Assumption 1.1 and Hölder's inequality that

$$
\begin{aligned}
& \left\langle A_{n}(v), v\right\rangle=\int_{\Omega}\left(|\nabla v|^{p}-\mu|\nabla v|^{q}-f(x, \rho * v, \nabla(\rho * v)) v\right) d x \\
& \geq\|\nabla v\|_{L^{p}\left(\Omega, \mathbb{R}^{N}\right)}^{p}-\mu|\Omega|^{\frac{p-q}{p}}\|\nabla v\|_{L^{p}\left(\Omega, \mathbb{R}^{N}\right)}^{q}-\|\sigma\|_{L^{r^{\prime}}(\Omega)}\|v\|_{L^{r}(\Omega)} \\
& -a_{1}\|\rho * v\|_{L^{p}\left(\mathbb{R}^{N}\right)}^{p-1}\|v\|_{L^{p}(\Omega)}-a_{2}\|\nabla(\rho * v)\|_{L^{p}\left(\mathbb{R}^{N}, \mathbb{R}^{N}\right)}^{p}\|v\|_{L^{p}(\Omega)}
\end{aligned}
$$

for all $v \in X_{n}$. Hereafter, we denote by $|\Omega|$ the Lebesgue measure of $\Omega$. Then (2.2), (2.4), and (2.1) lead to the estimate

$$
\begin{aligned}
& \left\langle A_{n}(v), v\right\rangle \geq\|\nabla v\|_{L^{p}\left(\Omega, \mathbb{R}^{N}\right)}^{p}-\mu|\Omega|^{\frac{p-q}{p}}\|\nabla v\|_{L^{p}\left(\Omega, \mathbb{R}^{N}\right)}^{q} \\
& -\|\sigma\|_{L^{r^{\prime}}(\Omega)}\|v\|_{L^{r}(\Omega)}-a_{1}\|\rho\|_{L^{1}\left(\mathbb{R}^{N}\right)}^{p-1}\|v\|_{L^{p}(\Omega)}^{p} \\
& -a_{2} N^{p-1}\|\rho\|_{L^{1}\left(\mathbb{R}^{N}\right)}^{p-1}\|\nabla v\|_{L^{p}\left(\Omega, \mathbb{R}^{N}\right)}^{p-1}\|v\|_{L^{p}(\Omega)} \\
& \geq\|\nabla v\|_{L^{p}\left(\Omega, \mathbb{R}^{N}\right)}^{p}-\mu|\Omega|^{\frac{p-q}{p}}\|\nabla v\|_{L^{p}\left(\Omega, \mathbb{R}^{N}\right)}^{q}-S_{r}\|\sigma\|_{L^{r^{\prime}(\Omega)}}\|\nabla v\|_{L^{p}\left(\Omega, \mathbb{R}^{N}\right)} \\
& -\left(a_{1} S_{p}^{p}\|\rho\|_{L^{1}\left(\mathbb{R}^{N}\right)}^{p-1}+a_{2} S_{p} N^{p-1}\|\rho\|_{L^{1}\left(\mathbb{R}^{N}\right)}^{p-1}\right)\|\nabla v\|_{L^{p}\left(\Omega, \mathbb{R}^{N}\right)}^{p}
\end{aligned}
$$

for all $v \in X_{n}$. Taking into account (1.4) (recall that $S_{p}=\lambda_{1, p}^{-\frac{1}{p}}$ ) and that $p>q>1$, the following estimate is true

$$
\left\langle A_{n}(v), v\right\rangle \geq 0 \text { whenever } v \in X_{n} \text { with }\|\nabla v\|_{L^{p}\left(\Omega, \mathbb{R}^{N}\right)}=R
$$


provided $R>0$ is sufficiently large. Then Lemma 2.1 yields the existence of $u_{n} \in X_{n}$ satisfying $A_{n}\left(u_{n}\right)=0$, that is, (3.3).

It remains to show that the sequence $\left\{u_{n}\right\}_{n \geq 1}$ is bounded in $W_{0}^{1, p}(\Omega)$. By inserting $v=u_{n} \in X_{n}$ in (3.4), we find that

$$
\begin{aligned}
& \left(1-\|\rho\|_{L^{1}\left(\mathbb{R}^{N}\right)}^{p-1}\left(a_{1} S_{p}^{p}+a_{2} S_{p} N^{p-1}\right)\right)\left\|\nabla u_{n}\right\|_{L^{p}\left(\Omega, \mathbb{R}^{N}\right)}^{p} \\
& \leq \mu|\Omega|^{\frac{p-q}{p}}\left\|\nabla u_{n}\right\|_{L^{p}\left(\Omega, \mathbb{R}^{N}\right)}^{q}+S_{r}\|\sigma\|_{L^{r^{\prime}}(\Omega)}\left\|\nabla u_{n}\right\|_{L^{p}\left(\Omega, \mathbb{R}^{N}\right)} .
\end{aligned}
$$

The desired conclusion is readily obtained from assumption (1.4) and the fact that $p>q>1$.

\subsection{Main result on the existence of a solution to problem (1.1)}

First, we show that the notions of generalized solution and weak solution coincide for problem (1.1) in the case where $\mu \leq 0$.

Lemma 3.3. Suppose that $\mu \leq 0$. For every $u \in W_{0}^{1, p}(\Omega)$, the following conditions are equivalent:

(i) $u$ is a weak solution to problem (1.1), that is, $u$ satisfies

$$
\left\langle-\Delta_{p} u+\mu \Delta_{q} u, v\right\rangle=\int_{\Omega} f(x, \rho * u(x), \nabla(\rho * u)(x)) v(x) d x
$$

for all $v \in W_{0}^{1, p}(\Omega)$

(ii) $u$ is a generalized solution to problem (1.1).

Proof. The implication (i) $\Rightarrow$ (ii) is immediate (take $u_{n}=u$ ) and actually does not require the condition that $\mu \leq 0$. Conversely, assume that $u$ is a generalized solution to problem (1.1), and let $\left\{u_{n}\right\}_{n \geq 1}$ be a sequence satisfying conditions (a)-(c) of the definition of generalized solution with respect to $u$. Using the monotonicity of the operator $-\Delta_{q}$ we note that

$$
\begin{aligned}
\left\langle-\Delta_{p} u_{n}, u_{n}-u\right\rangle & \leq\left\langle-\Delta_{p} u_{n}, u_{n}-u\right\rangle-\mu\left\langle-\Delta_{q} u_{n}+\Delta_{q} u, u_{n}-u\right\rangle \\
& =\left\langle-\Delta_{p} u_{n}+\mu \Delta_{q} u_{n}, u_{n}-u\right\rangle-\mu\left\langle\Delta_{q} u, u_{n}-u\right\rangle .
\end{aligned}
$$

By (a) and (c), this leads to

$$
\limsup _{n \rightarrow \infty}\left\langle-\Delta_{p} u_{n}, u_{n}-u\right\rangle \leq 0 .
$$

Then we are able to conclude the strong convergence $u_{n} \rightarrow u$ in $W^{1, p}(\Omega)$ (see, e.g., [7, Proposition 2.72]). By Lemma 3.1, this implies that $A\left(u_{n}\right) \rightarrow A(u)$ in $W^{-1, p^{\prime}}(\Omega)$, where $A: W_{0}^{1, p}(\Omega) \rightarrow W^{-1, p^{\prime}}(\Omega)$ is the operator defined in (3.1). In view of condition (b) of the definition of generalized solution, this yields $A(u)=0$, which precisely means that $u$ is a weak solution to problem (1.1).

We can now state our main result.

Theorem 3.4. Suppose that Assumption 1.1 holds. Then there exists a generalized solution to problem (1.1). In particular, if $\mu \leq 0$, there exists a weak solution to problem (1.1). 
Proof. Consider the sequence $\left\{u_{n}\right\}_{n \geq 1} \subset W_{0}^{1, p}(\Omega)$ constructed in Proposition 3.2. As asserted therein, this sequence is bounded in $W_{0}^{1, p}(\Omega)$. In view of the reflexivity of the space $W_{0}^{1, p}(\Omega)$, we can pass to a subsequence still denoted by $\left\{u_{n}\right\}_{n \geq 1}$ such that

$$
u_{n} \rightarrow u \text { in } W_{0}^{1, p}(\Omega)
$$

with some $u \in W_{0}^{1, p}(\Omega)$. Moreover, since the sequence $\left\{u_{n}\right\}_{n \geq 1}$ is bounded in $W_{0}^{1, p}(\Omega)$, invoking the continuity of the operator in (3.2), we have that

$$
\text { the sequence }\left\{f\left(\cdot, \rho * u_{n}, \nabla\left(\rho * u_{n}\right)\right)\right\}_{n \geq 1} \text { is bounded in } L^{p^{\prime}}(\Omega) \text {. }
$$

On the basis of the reflexivity of $W^{-1, p^{\prime}}(\Omega)$, we can assume that

$$
-\Delta_{p} u_{n}+\mu \Delta_{q} u_{n}-f\left(\cdot, \rho * u_{n}, \nabla\left(\rho * u_{n}\right)\right) \rightarrow \eta \text { in } W^{-1, p^{\prime}}(\Omega)
$$

with some $\eta \in W^{-1, p^{\prime}}(\Omega)$.

Now let $v \in \bigcup_{n \geq 1} X_{n}$. Fix an integer $m \geq 1$ such that $v \in X_{m}$. Proposition 3.2 provides that (3.3) holds for all $n \geq m$. Letting $n \rightarrow \infty$ in (3.3), by means of (3.7) we get

$$
\langle\eta, v\rangle=0 \text { for all } v \in \bigcup_{n \geq 1} X_{n} .
$$

By the density of $\bigcup_{n \geq 1} X_{n}$ in $W_{0}^{1, p}(\Omega)$ (see (iii) in the definition of Galerkin basis in Section 2.1), it turns out that $\eta=0$. Therefore, (3.7) renders

$$
-\Delta_{p} u_{n}+\mu \Delta_{q} u_{n}-f\left(\cdot, \rho * u_{n}, \nabla\left(\rho * u_{n}\right)\right) \rightarrow 0 \text { in } W^{-1, p^{\prime}}(\Omega) .
$$

Next, setting $v=u_{n}$ in (3.3), we obtain

$$
\left\langle-\Delta_{p} u_{n}+\mu \Delta_{q} u_{n}, u_{n}\right\rangle-\int_{\Omega} f\left(x, \rho * u_{n}, \nabla\left(\rho * u_{n}\right)\right) u_{n} d x=0
$$

for all $n \geq 1$, while (3.8) gives

$$
\left\langle-\Delta_{p} u_{n}+\mu \Delta_{q} u_{n}, u\right\rangle-\int_{\Omega} f\left(x, \rho * u_{n}, \nabla\left(\rho * u_{n}\right)\right) u d x \rightarrow 0
$$

as $n \rightarrow \infty$. Altogether, (3.9) and (3.10) yield

$$
\left\langle-\Delta_{p} u_{n}+\mu \Delta_{q} u_{n}, u_{n}-u\right\rangle-\int_{\Omega} f\left(x, \rho * u_{n}, \nabla\left(\rho * u_{n}\right)\right)\left(u_{n}-u\right) d x \rightarrow 0
$$

as $n \rightarrow \infty$. Moreover, from (3.5), Rellich-Kondrachov compact embedding theorem which ensures that $u_{n} \rightarrow u$ strongly in $L^{p}(\Omega)$, and (3.6), we derive that

$$
\lim _{n \rightarrow \infty} \int_{\Omega} f\left(x, \rho * u_{n}, \nabla\left(\rho * u_{n}\right)\right)\left(u_{n}-u\right) d x=0 .
$$

Inserting (3.12) into (3.11) enables us to assert

$$
\lim _{n \rightarrow \infty}\left\langle-\Delta_{p} u_{n}+\mu \Delta_{q} u_{n}, u_{n}-u\right\rangle=0 .
$$

At this point we can notice that (3.5), (3.8), and (3.13) are just the conditions (a), (b), and (c) expressing that $u \in W_{0}^{1, p}(\Omega)$ is a generalized solution to problem (1.1), which proves the first assertion in the theorem. The last assertion in the theorem is a consequence of Lemma 3.3. 
Acknowledgement. The authors are grateful to Dr. Lucas Fresse for important remarks.

\section{References}

[1] Brezis, H., Functional Analysis, Sobolev Spaces and Partial Differential Equations, Universitext, Springer, New York, 2011.

[2] Krasnoselskii, M.K., Topological Methods in the Theory of Nonlinear Integral Equations, Pergamon Press, New York, 1964.

[3] Liu, Z., Livrea, R., Motreanu, D., Zeng, S., Variational differential inclusions without ellipticity condition, Electron. J. Qual. Theory Differ. Equ., Paper No. 43 (2020), 17 pp.

[4] Motreanu, D., Nonlinear Differential Problems with Smooth and Nonsmooth Constraints, Academic Press, London, 2018.

[5] Motreanu, D., Quasilinear Dirichlet problems with competing operators and convection, Open Math., 18(2020), 1510-1517.

[6] Motreanu D., Motreanu, V.V., Non-variational elliptic equations involving $(p, q)$ Laplacian, convection and convolution, Pure Appl. Funct. Anal., 5(2020), 1205-1215.

[7] Motreanu, D., Motreanu, V.V., Papageorgiou, N.S., Topological and Variational Methods with Applications to Nonlinear Boundary Value Problems, Springer, New York, 2014.

[8] Showalter, R.E., Monotone Operators in Banach Space and Nonlinear Partial Differential Equations, Mathematical Surveys and Monographs, vol. 49, American Mathematical Society, Providence, RI, 1997.

Dumitru Motreanu

Département de Mathématiques,

Université de Perpignan,

66860 Perpignan, France

e-mail: motreanu@univ-perp.fr

Viorica Venera Motreanu

Collège Jean Moulin,

14 rue Jean Moulin,

54510 Tomblaine, France

e-mail: vmotreanu@gmail.com 